\title{
Impact of Stretch and Heat Loss on Flame Stabilization in a Lean Premixed Flame approaching Blow-off
}

\author{
Pier Carlo Nassini ${ }^{\mathrm{a}, *}$, Daniele Pampaloni ${ }^{\mathrm{a}}$, Antonio Andreini ${ }^{\mathrm{a}}$ \\ ${ }^{a}$ Department of Industrial Engineering, University of Florence, Via di S. Marta, 3, Firenze 50139, Italy
}

\begin{abstract}
The accurate prediction of the turbulent combustion process in lean burn flames is of primary importance in the design of gas turbine low-emission combustors. In this framework, the correct account for high strain levels, combined with the heat loss of the flame, by numerical tools is of high technical relevance in order to improve the operational flexibility while reducing emissions. In fact, in high Reynolds lean combustion modelling, the quenching effects due to flame front distortion are expected to govern flame behaviour. The present work presents an assessment of the modelling strategies to introduce the stretch effects on the flame in Flamelet Generated Manifold (FGM) model, in both the framework of Reynolds-Averaged Navier-Stokes (RANS) and Large-Eddy Simulation (LES). At this purpose a premixed swirl burner experimentally studied at Cambridge University was chosen, consisting of a strongly swirling, confined natural gas flame. Results highlight that LES-FGM, coupled with an extended Turbulence Flame Closure model (TFC), succeeds in predicting the main characteristics of the flame at different operating conditions approaching blow-off, thus representing a valid tool to investigate lean burn flames in such context.
\end{abstract}

(C) 2018 The Authors. Published by Elsevier Ltd.

This is an open access article under the CC BY-NC-ND license (https://creativecommons.org/licenses/by-nc-nd/4.0/)

Selection and peer-review under responsibility of the scientific committee of the 73rd Conference of the Italian Thermal Machines Engineering Association (ATI 2018).

Keywords: Gas turbine; Combustion; Blow-off; Stretch; Swirl flame; Premixed; CFD; LES; FGM.

\section{Introduction}

During the years, the regulation for pollutant emissions has become more and more strict, leading gas turbine manufacturers to develop combustors with increasingly low levels of emissions. To meet these law restrictions, modern combustors rely on Lean Premixed (LP) combustion and operate near the lean blow-off limit. However, near the lean limit, strong instabilities and global extinction can represent a serious problem for the designers.

In this framework, Computational Fluid Dynamics (CFD) has become a powerful tool, able to provide detailed and multi-scale information about combustion processes. Reynolds-Averaged Navier-Stokes equations (RANS), due

\footnotetext{
${ }^{*}$ Corresponding author. Tel.: +39-055-275-8772.

E-mail address: piercarlo.nassini@htc.de.unifi.it
} 
to the reduced computational effort, have become the widespread standard in the industrial framework but are often unable or insufficient to properly characterize the effects of turbulent mixing [7]. Using Large Eddy Simulation (LES), instead, the modelling error could be reduced by directly solving part of the turbulent energy cascade, thus leading to an improved prediction of the turbulent flow field.

Regarding the combustion modelling, in order to reproduce the complex topology and the stabilization mechanism of lean burn flames and to properly characterize pollutant emissions, approaches characterized by a detailed description of the chemical kinetics are required. In the field of combustion engineering, the Flamelet Generated Manifold (FGM) model is very appreciated for its good accuracy and versatility, despite the low computational cost [13]. However, the correct account for high strain levels, combined with the heat loss of the flame, is mandatory in high Reynolds lean combustion modelling, since the quenching effects due to flame front distortion are expected to govern flame parameters like shape, emissions and blow-off. Different strategies could be used to introduce the stretch effects on the flame. In this work, the capabilities of such models to provide reliable predictions of the turbulent combustion process in lean flames are assessed. At this purpose the premixed swirl burner experimentally studied at Cambridge University [5] was chosen, consisting of a strongly swirling, confined natural gas flame. Both RANS and LES simulations with the influence of flame stretch are carried out and validated against experimental measurements for different operating conditions approaching blow-off.

\section{Nomenclature}

$\begin{array}{ll}A & \text { Zimont TFC constant } \\ a & \text { total strain } \\ \alpha & \text { thermal diffusivity } \\ B & \text { constant in critical strain correlation } \\ \phi & \text { equivalence ratio } \\ K a & \text { stretch Karlovitz number } \\ M a & \text { Markstein number } \\ \varphi & \text { heat loss parameter } \\ g_{c r} & \text { critical strain } \\ S_{c} & \text { consumption speed } \\ S_{l}^{0} & \text { unstretched laminar flame speed } \\ S_{N} & \text { swirl number } \\ U_{b} & \text { axial bulk velocity }\end{array}$

\section{Description of test case}

The swirl burner investigated in this work has been studied experimentally at the University of Cambridge [4]. The fully premixed methane-air configuration, shown in Figure 1, is investigated. It consists of a $350 \mathrm{~mm}$ long annular duct with external diameter of $37 \mathrm{~mm}$, fitted with a centred conical bluff body of diameter $D_{b}=25 \mathrm{~mm}$ that gives a 50\% blockage ratio. The burner feeds a squared-section combustion chamber enclosed by synthetic optical quartz walls with a width of $95 \mathrm{~mm}$ and length of $150 \mathrm{~mm}$. The outlet of the chamber is open to the atmosphere. A premixed methane-air mixture at $288 \mathrm{~K}$ flows in the annulus and gets swirl motion through a static axial swirler with six blades oriented at $60^{\circ}$ with respect to the flow axis. The swirl number, estimated with the expression derived by Beer and Chigier [2] is $S_{N}=1.23$. The experimental measurements on premixed flame conducted by Kariuki [8] investigated both stable and approaching blow-off operating conditions, namely P1 - 3, as reported in Table 1 . The P1 condition corresponds to a stable flame that can be used as reference; P2 is a near blow-off condition that should exhibit some features probably due to local extinctions; P3 condition is just prior LBO event, since a further reduction of equivalence ratio leads to the complete extinction of the flame. 
Table 1. Flame conditions investigated, derived from [5].

\begin{tabular}{|c|c|c|c|c|c|c|}
\hline Name & Case & $\boldsymbol{U}_{\boldsymbol{b}}[\mathrm{m} / \mathrm{s}]$ & $\phi$ & $\phi / \phi_{B O}$ & $\dot{\boldsymbol{m}}[\mathrm{g} / \mathrm{s}]$ & $Z$ \\
\hline $\mathrm{C} 1$ & Cold flow & 14.3 & - & - & 9.83 & 0 \\
\hline P1 & Stable flame & 19.9 & 0.70 & $124 \%$ & 13.48 & 0.0391 \\
\hline $\mathrm{P} 2$ & Near blow-off flame & 19.7 & 0.61 & $108 \%$ & 13.37 & 0.0343 \\
\hline P3 & Just prior blow-off flame & 19.6 & 0.57 & $101 \%$ & 13.32 & 0.0321 \\
\hline
\end{tabular}

\section{Numerical setup}

The simulations are carried out using the commercial CFD code ANSYS Fluent v16.1 [1]. Both RANS and LES simulations have been carried out. The computational domain used for the simulations is represented in Figure 1. Three parts can be identified: (1) an annular burner fitted with an axial swirler and a conical bluff body, (2) a squaredsection combustion chamber and (3) an hemispherical far-field, added to simulate the open atmospheric discharge and, at the same time, to reduce acoustic waves reflection on outlet surface. The domain is discretized in approximately

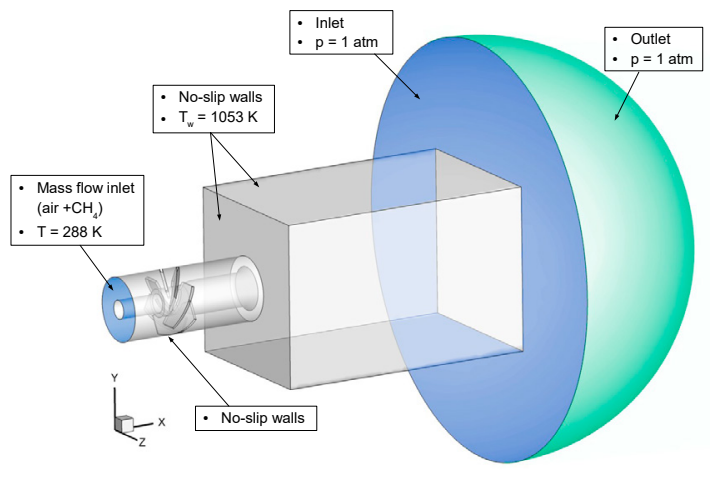

(a)

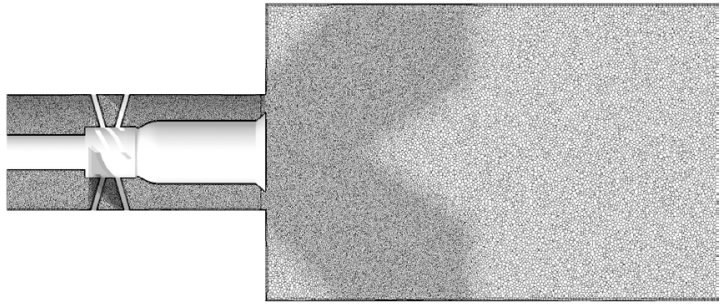

(b)

Fig. 1. Computational domain with main boundary conditions (a) and section of the mesh in the $x y$ plane (b).

7 million polyhedral cells, as shown in Figure 1. The characteristic size of polyhedral cells is about $0.5 \mathrm{~mm}$ in the burner and $1 \mathrm{~mm}$ in the chamber, with a refinement of $0.5 \mathrm{~mm}$ that extends inside the chamber for $65 \mathrm{~mm}$ in a region with high mixing and shear, as reported by Cavaliere [4]. In the far-field zone the cell dimension gradually raises since there is no need to resolve detailed flow structures and bigger cells results in a numerical damping of perturbations reflected on outlet surface. The imposed boundary conditions are summarised in Table 1 and Figure 1 for each test point. For the RANS simulations a turbulence intensity of 5\% and the relative hydraulic diameter is imposed at the inlets and outlets while in LES no artificial turbulence is introduced through these surfaces.

Within the RANS framework, the effects of turbulence are modelled with the $k-\epsilon$ two-equation model including the realizability correction [10]. In LES simulations, the sub-grid turbulence is modelled with the Dynamic SmagorinskyLilly formulation [9]. Concerning combustion modelling, the Flamelet Generated Manifold (FGM) model implemented in Fluent [1] has been used. In FGM a multi-dimensional manifold is generated solving a set of laminar adiabatic one-dimensional flamelets and describing the chemical state and reaction progress space only as function of few control variables, such as mixture fraction $\mathrm{Z}$ and normalized progress variable $\mathrm{c}$. Flamelet equations have been solved using the Fluent built-in CHEMKIN solver and exploiting the Gas Research Institute reaction mechanism GRImech 3.0 for $\mathrm{CH}_{4}$, with 325 reactions and 53 species. Finally, the source term of progress variable has been modelled in the present work with either the Zimont Turbulent Flame Closure (TFC) or an extended TFC model able to deal with the effects of both flame strecth and heat loss. The extension of TFC model is implemented in Fluent following the approach of Tay-Wo-Chong et al. [11]. The model relies on the empirical correlation (1) for the flame consumption speed developed by Tay-Wo-Chong et al. [12] from 1D calculations of strained lean premixed methane-air flamelets 
in asymmetric counterflow configuration at atmospheric pressure:

$$
S_{c}=S_{l}^{0} \exp [-K a(M a+\varphi)]
$$

The consumption speed is expressed reducing the unstretched laminar flame speed $S_{l}^{0}$ with the effects of (1) the strain induced by the turbulence and the mean or resolved flow, (2) the heat loss respect to the adiabatic temperature of local products and (3) the Markstein number Ma at adiabatic conditions. The first two effects enters respectively in the stretch Karlovitz number $K a$ and in the heat loss parameter $\varphi$. For more details about the model implementation see the formulation of Tay-Wo-Chong et al. $[12,11]$.

In the LES calculations a time step of $1 \times 10^{-5} \mathrm{~s}$ is applied so that the maximum Courant number in the chamber is lower than 0.6 .

\section{Results}

To validate the numerical setup and the computational domain, both the non-reacting and the reacting flow fields are analysed and compared against Laser Doppler Velocimetry (LDV) measurements by Cavaliere [4].

\subsection{Non-reacting flow field}

A RANS simulation is carried out at the same conditions C1 used for the measurements, summarized in Table 1. In Figure 2 the axial velocity flow field on a longitudinal plane is shown. The swirling jet exits the burner forming a conical shape with an angle of roughly $60^{\circ}$ and it impinges the wall of the chamber at a distance of about $x / D_{b}=1.5$ from the base. The chamber is almost completely occupied by the large inner recirculation zone (IRZ) that extends beyond the chamber. The simulated velocity field is compared with experimental data at the different streamwise positions $x=8,13,18,33 \mathrm{~mm}$. The comparison shows that the accuracy of the mean velocity prediction is very satisfatory at every streamwise position, as showed in Figure 2 for the first and last location. The extension of the recirculation zone is correctly reproduced as well as the reverse flow velocity.

\subsection{Reacting flow-field}

Within the Flamelet Generated Manifold approach, the modelling choice that most influences the flame structure is the closure of progress variable source term. In the present work, the Zimont TFC [14] and the extended TFC models
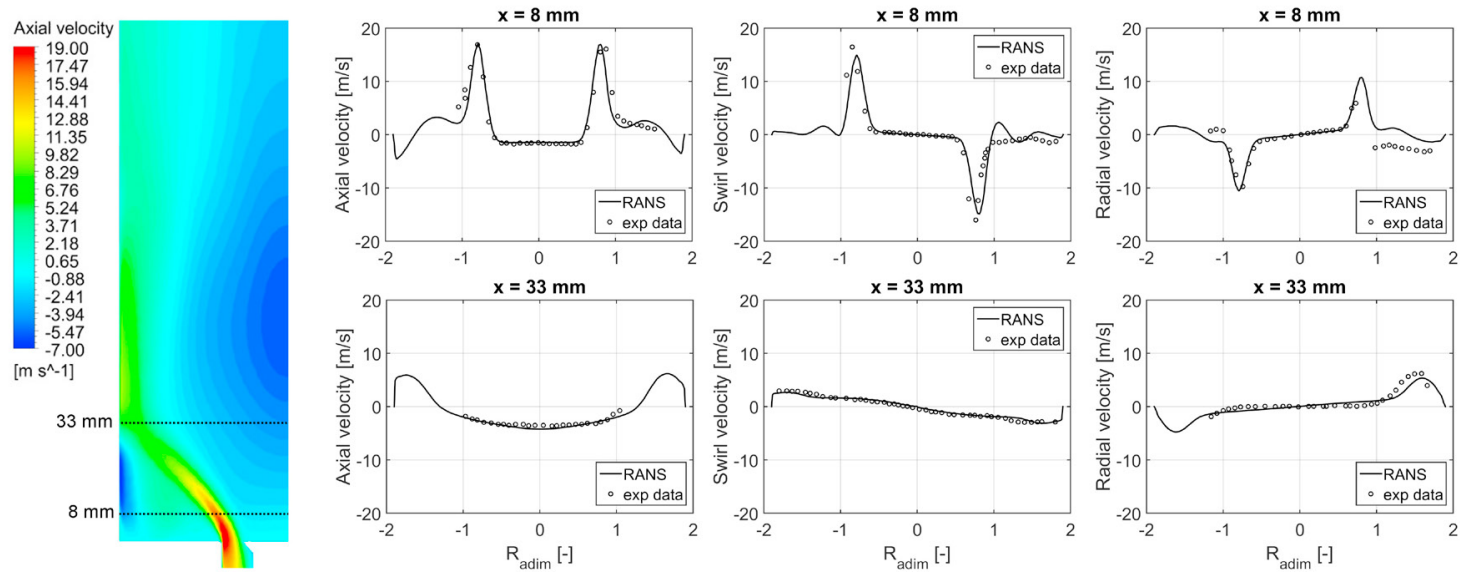

Fig. 2. Axial velocity field on longitudinal $x y$ plane (left); velocity profiles along radial direction at $8 \mathrm{~mm}$ and $33 \mathrm{~mm}$ inside the chamber, compared with LDV data [4]. Isothermal condition C1. 
are tested and compared for three different flame conditions, reported in Table 1. In order to be able to predict with reasonable accuracy the inception of blow-off, the desired setup should be able to capture not only the single flame conditions, but also the flame evolution approaching lean blow-off. To do that, the setup should be validated against experimental data over all the flame conditions, and not to be adjusted as the equivalence ratio is decreased. At first, the results of RANS simulations with different closures are presented in the following sections for the three flame conditions P1, P2 and P3. Then, the optimized setup will be applied and validated in the LES framework.

\subsubsection{Zimont TFC model}

The normalized $\mathrm{OH}$ distributions that result from the simulation with Zimont Turbulent Flame Closure are presented in Figure 3, in comparison with Kariuki [8]'s OH-PLIF measurements. From the obtained OH distribution the

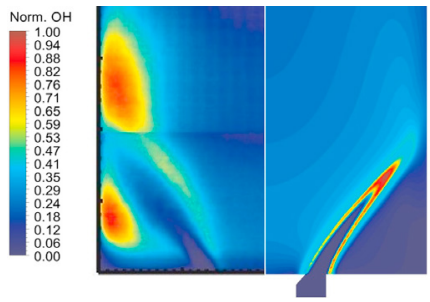

(a) P1

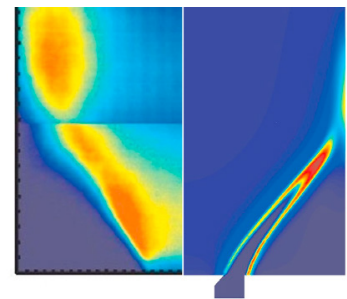

(b) P2

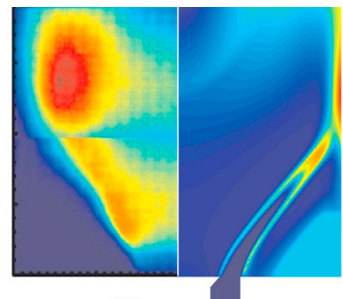

(c) P3

Fig. 3. Comparison of normalized $\mathrm{OH}$ mass fraction on $x y$ plane between experiments (left side) and RANS simulations with Zimont TFC (right side) for the three flame conditions.

reaction appears very fast. In P1 and P2 conditions the flame brush is thin and becomes wider in front of the swirling jet, consuming all the mixture much before the walls. The lower equivalence ratio moves slightly downstream the flame, but the flame shape does not change qualitatively. For the condition just prior blow-off (P3) a noticeable variation of $\mathrm{OH}$ concentration can be observed. The reactivity in both internal and external shear layer of the jet slows down, as well as at the tip of fresh mixture stream. The leaner conditions delay the completion of combustion, so that the peak value in $\mathrm{OH}$ concentration is registered along the wall. Concerning the comparison with experimental measurements, the flame evolution approaching blow-off is not well captured: the flame speed seems to be overestimated and the radical change between P1 and P2 condition is not captured. In these conditions, the correct modelling of the flame front stretch, able to locally quench the reaction, can improve the result accuracy. This fact was acknowledged by Zimont itself and he introduced the stretch factor $G$ formulated by Bray [3] to account for the quenching of flamelets, through the specification of a critical strain $g_{c r}$ as threshold between totally quenched and unquenched flamelets. It is possible estimate a value for $g_{c r}$ through the correlation [1, 6]:

$$
g_{c r}=\frac{B S_{l}^{0^{2}}}{\alpha}
$$

Different values of the constant $B$ were tested, from 0.5 to 0.1 (corresponding to $g_{c r}$ within the range 2600-13000 1/s), to asses their impact on the flame. This approach allows an automatic reduction of $g_{c r}$ i.e. an enhanced sensitivity to stretch of leaner flamelets which have lower values of laminar flame speed. In Figures 4 the values of $\mathrm{OH}$ mass fraction are showed in function of the critical strain $g_{c r}$. Reducing the constant B to 0.25 , i.e. halving the critical strain, the fresh mixture is not consumed completely before reaching the walls of the chamber. The $\mathrm{OH}$ mass fraction 4(b) shows two distinct peaks along the wall in both corner recirculation zone (CRZ) and just downstream the impingement point. In addiction, the shear layers of the swirling jet do not presents the high $\mathrm{OH}$ concentrations of the previous cases, probably because here the levels of strain are above the threshold $g_{c r}$. In the case with the lower constant $B=0.1$ the flame is able to stabilize only in the region near the zero-velocity streamline where the strain is lower. From the comparison to the mean OH-PLIF experiments by Kariuki [8] it is clear that the simulation with a constant $B=0.25$ predicts with reasonable accuracy the stable flame, in particular with the characteristic two separated peaks of $\mathrm{OH}$ near 


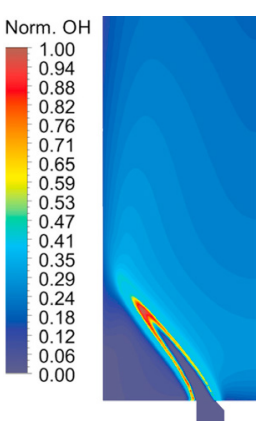

(a) $B=0.5$

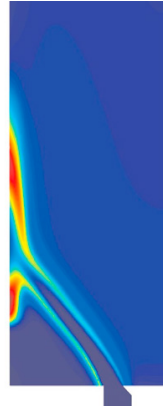

(b) $B=0.25$

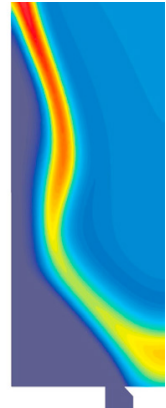

(c) $B=0.1$

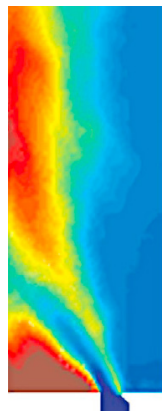

(d) $B=0.25$ (LES)

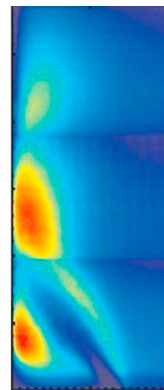

(e) OH-PLIF

Fig. 4. Normalized OH mass fraction on $x y$ plane with different values of $g_{c r}$. RANS results (a)-(c); time averaged LES results (d); OH-PLIF [5] (e).

the walls (Figure 3). The same constant of $B=0.25$ was therefore applied to LES 3. As in RANS framework, with respect to the default setup with $G=1$, the reduction of $g_{c r}$ has a great influence on the solution. LES predicts a much wider flame brush than RANS simulation, but the key features are maintained with strong OH concentration in CRZ and downstream the point of impingement near the wall. Despite some discrepancies, the stretch factor $(B=0.25)$ can improve substantially the solution for the stable flame condition (P1) with respect to the default model, both in RANS and LES. However, applying this FGM setup of Zimont TFC with $B=0.25$ to P3 condition, i.e. just prior blow-off $(\phi=0.57)$, the flame cannot stabilize and blows off during the numerical transitory in RANS simulation. In fact, the stretch effect is overestimated near LBO using the correlation (2), probably for its strong dependence on laminar flame speed (see (2)). Excluding the correlation, a value for $g_{c r}$ could be imposed directly, but this would require a tuning over all the different conditions in a particular test case. Since the objective of this analysis is to find out one setup valid for all the three flame conditions investigated, the Zimont TFC model with the stretch factor is discarded.

\subsubsection{Extended TFC model}

As highlighted in the previous results, the stretch factor formulation for Zimont TFC is not able to predict correctly the effects that the deformation of flame front has on the turbulent flame speed. For this reason, the extended TFC model is specifically exploited as a different strategy to include these effects. All the three flame conditions P1 to P3 are investigated with RANS simulations, and the results are presented here.

In Figure 5 the normalized $\mathrm{OH}$ mass fraction on $x y$ plane obtained with RANS extension of Zimont are compared to the OH-PLIF results by Kariuki [8] for the three flame conditions P1 to P3. Even if some features of the real flame are not captured by the simulations, the extension shows an improvement of accuracy with respect to the default model. As far as concern the stable flame P1, the RANS simulation does not show the OH concentration on internal shear layer of the jet captured by PLIF measurements. This differences could be related to the reduced diffusion predicted by the steady simulation. Other key features are actually found in the RANS field, such as the double $\mathrm{OH}$ peak just downstream the impingement point of the jet on the wall and the high $\mathrm{OH}$ in the corner recirculation.

Approaching blow-off (P2-P3) the simulations well predict the absence of $\mathrm{OH}$ radicals in the corners and the shape of the jet, but for $\mathrm{P} 2$ flame the zone above the bluff body and the internal shear layer does not contain almost any $\mathrm{OH}$ radical, while in $\mathrm{P} 3$ condition here it is moderately overestimated.

The extended TFC model is applied to LES with the objective to improve the accuracy of the solutions obtained in extended RANS simulation. The results of normalized mean $\mathrm{OH}$ mass fraction are reported in Figure 6 for the considered flame conditions P1, P2 and P3. The coupling between LES and TFC extension shows an improvement of accuracy with respect to the RANS framework. In particular P2 shows a well predicted $\mathrm{OH}$ distribution, concerning the main peak shape and position comparable to the measurements. Nevertheless, the underestimation of $\mathrm{OH}$ emission within the internal shear layer persists also in this case and a non-negligible $\mathrm{OH}$ mass fraction, not present in PLIF measurement, is predicted in the corner zone. Despite the differences between predicted and measured $\mathrm{OH}$ distributions, the overall prediction of all the different flame operating conditions is satisfactory. It is important to stress that the same LES-FGM setup with extended TFC model is used in every condition without changing. This setup is able 


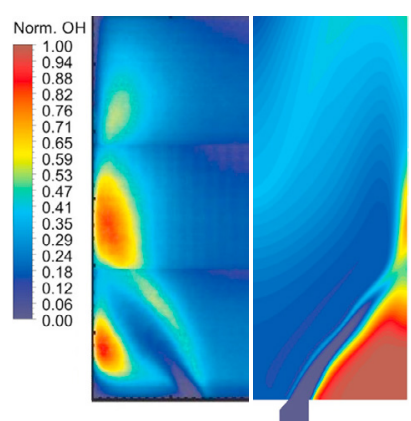

(a) (b)

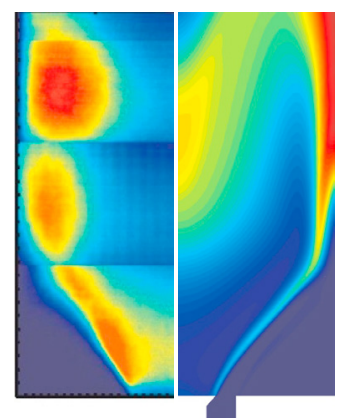

(c) (d)

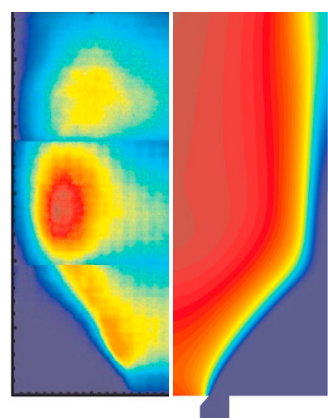

(e) (f)

Fig. 5. Comparison of OH-PLIF (a)(c)(e) [8] and normalized OH mass fraction predicted by RANS simulation with Zimont TFC extension (b) (d) (f) in the conditions P1, P2 and P3 (from left to right).

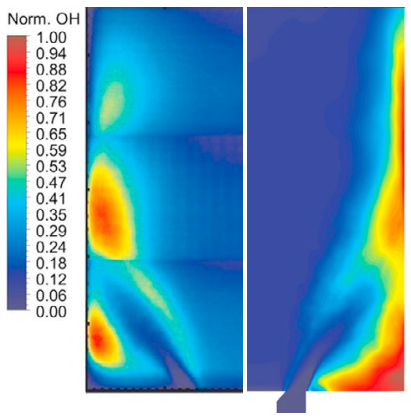

(a) (b)

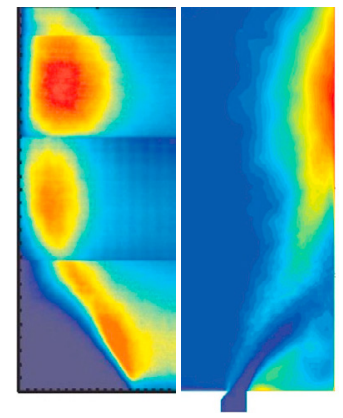

(c) (d)

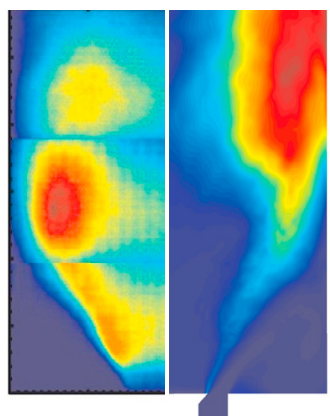

(e) (f)

Fig. 6. Comparison of OH-PLIF (a)(c)(e) [8] and normalized mean $\mathrm{OH}$ mass fraction predicted by LES with Zimont TFC extension and $A=1$ (b)(d)(f) in the conditions P1, P2 and P3 (from left to right).

to capture the main evolution of flame characteristics approaching blow off, with the progressive reduction of $\mathrm{OH}$ in the corner and the thickening of high emission region approaching blow-off.

The consumption speed is the key parameter of the extended TFC model, so it is important to analyse its field. In Figure 7(a) is reported an instantaneous distribution of the consumption speed $S_{c}$ normalized with respect to the unstretched laminar flame speed $S_{l}^{0}$ to highlight the effect of the flame strecth and heat loss correction. The black and red lines on Figure 7(a) are isolevels of total strain $a=30001 / \mathrm{s}$ and heat loss $\varphi=0.2$ respectively. Strain higher than $3000 \mathrm{1} / \mathrm{s}$ reduces importantly the laminar flame speed especially in the shear layers of the jet and in the second half of the chamber. The heat loss is mainly confined near the walls and in the corner where it presents high values which amplify the strain effect over 10 times.

\section{Conclusions}

In this work a premixed strongly-swirled confined flame is investigated as blow-off is approached. Different strategies to introduce the stretch effects on the flame in FGM model are tested and compared with respect to detailed experimental measurements. The main outcomes of the present investigation are the following:

- The standard Zimont TFC model overestimates the reaction rate because of an incorrect turbulent flame speed. The low accuracy of the model is judged related to the missed modelling of the stretch of the flame front, locally able to quench the reaction;

- The stretch factor $G$ formulation can improve Zimont TFC results but is not suitable to simulate the flame evolution approaching blow-off, as it requires a tuning of the constant $B$ over all the different conditions; 


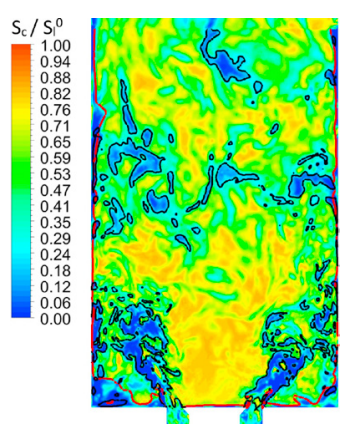

(a) Consumption speed $S_{c} / S_{l}^{0}$

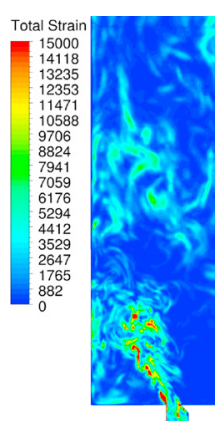

(b) Total strain $a$

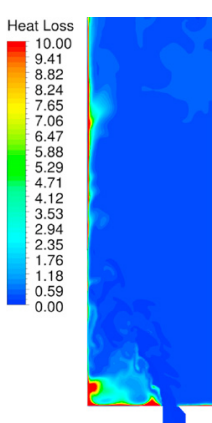

(c) Heat loss $\varphi$

Fig. 7. (a) Instantaneous consumption speed $S_{c}$ relative to unstretched laminar flame speed $S_{l}^{0}$. Black and white lines represent the isolevels of strain $\tilde{a}=3000 / s$ and heat $\operatorname{loss} \varphi=2$ respectively; (b) Total strain distribution; (c) Heat loss parameter. Stable flame P1, $x y$ plane.

- The extended TFC model includes specifically the physical effects of flame front stretching and thus allows a better prediction of the turbulence-chemistry interaction. This interaction becomes fundamental near the lower flammability limit since it is one of the main phenomena that trigger the global extinction. The model is validated over the three flame conditions within both RANS and LES framework. Despite some differences between predicted and measured $\mathrm{OH}$ distributions, the overall prediction of all the different flame operating conditions within the LES framework is satisfactory, showing the ability of the model to capture the main evolution of flame approaching blow off.

\section{Acknowledgements}

The authors would like to thank Prof Epaminondas Mastorakos and Dr. Andrea Giusti for the geometry and the useful publications provided.

\section{References}

[1] ANSYS, 2014. Fluent 16 Theory Guide.

[2] Beer, J.M., Chigier, N.A., 1972. Combustion aerodynamics. Applied Science, 100-146.

[3] Bray, K.N.C., 1987. Complex Chemical Reaction Systems. Mathematical Modelling and Simulation. Springer Verlag.

[4] Cavaliere, D.E., 2013. Blowoff in Gas Turbine Combustors. Ph.D. thesis. University of Cambridge.

[5] Cavaliere, D.E., Kariuki, J., Mastorakos, E., 2013. A Comparison of the Blow-Off Behaviour of Swirl-Stabilized Premixed , Non-Premixed and Spray Flames. Flow Turbulence Combustion, 347-372.

[6] Flohr, P., Pitsch, H., 2000. A turbulent flame speed closure model for LES of industrial burner flows. Center for Turbulence Research, Proceedings of the summer program , 169-179.

[7] Gicquel, L.Y.M., Staffelbach, G., Poinsot, T., 2013. Large Eddy Simulations of gaseous flames in gas turbine combustion chambers. Progress in Energy and Combustion Science 38, 782-817.

[8] Kariuki, J., 2012. Turbulent premixed flame stabilization and blow-off. Ph.D. thesis. University of Cambridge.

[9] Lilly, D.K., 1992. A Proposed Modification of the Germano-Subgrid-Scale Closure Method. Physics of Fluids A 4, $633-635$.

[10] Shih, T.H., Liou, W.W., Shabbir, A., Yang, Z., Zhu, J., 1995. A New - Eddy-Viscosity Model for High Reynolds Number Turbulent Flows Model Development and Validation. Computers Fluids. 24, 227-238.

[11] Tay-Wo-Chong, L., Scarpato, A., Polifke, W., 2017. LES Combustion Model with Stretch and Heat Loss Effects for Prediction of Premix Flame Characteristics and Dynamics, in: Proceedings of ASME Turbo Expo 2017: Turbomachinery Technical Conference and Exposition, pp. $1-12$.

[12] Tay-Wo-Chong, L., Zellhuber, M., Komarek, T., Im, H.G., Polifke, W., 2016. Combined Influence of Strain and Heat Loss on Turbulent Premixed Flame Stabilization. Flow, Turbulence and Combustion, 263-294.

[13] Van Oijen, J.A., Donini, A., Bastiaans, R.J.M., ten Thije Boonkkamp, J., de Goey, L., 2016. State-of-the-art in premixed combustion modeling using flamelet generated manifolds. Progress in Energy and Combustion Science 57, 30-74.

[14] Zimont, V., Polifke, W., Bettelini, M., Weisenstein, W., 1997. An efficient computational model for premixed turbulent combusiton at high reynolds numbers based on a turbulent flame speed closure. International Gas Turbine \& Aeroengine Congress \& Exhibition , 1-10. 